\title{
Noncoercive Perturbed Densely Defined Operators and Application to Parabolic Problems
}

\author{
Teffera M. Asfaw \\ Department of Mathematics, Virginia Polytechnic Institute and State University, Blacksburg, VA 24061, USA \\ Correspondence should be addressed to Teffera M. Asfaw; tefferam@yahoo.com
}

Received 30 June 2015; Accepted 9 August 2015

Academic Editor: Naseer Shahzad

Copyright (C) 2015 Teffera M. Asfaw. This is an open access article distributed under the Creative Commons Attribution License, which permits unrestricted use, distribution, and reproduction in any medium, provided the original work is properly cited.

\begin{abstract}
Let $X$ be a real locally uniformly convex reflexive separable Banach space with locally uniformly convex dual space $X^{*}$. Let $T$ : $X \supseteq D(T) \rightarrow 2^{X^{*}}$ be maximal monotone and $S: X \supseteq D(S) \rightarrow X^{*}$ quasibounded generalized pseudomonotone such that there exists a real reflexive separable Banach space $W \subset D(S)$, dense and continuously embedded in $X$. Assume, further, that there exists $d \geq 0$ such that $\left\langle v^{*}+S x, x\right\rangle \geq-d\|x\|^{2}$ for all $x \in D(T) \cap D(S)$ and $v^{*} \in T x$. New surjectivity results are given for noncoercive, not everywhere defined, and possibly unbounded operators of the type $T+S$. A partial positive answer for Nirenberg's problem on surjectivity of expansive mapping is provided. Leray-Schauder degree is applied employing the method of elliptic superregularization. A new characterization of linear maximal monotone operator $L: X \supseteq D(L) \rightarrow X^{*}$ is given as a result of surjectivity of $L+S$, where $S$ is of type $(M)$ with respect to $L$. These results improve the corresponding theory for noncoercive and not everywhere defined operators of pseudomonotone type. In the last section, an example is provided addressing existence of weak solution in $X=L^{p}\left(0, T ; W_{0}^{1, p}(\Omega)\right)$ of a nonlinear parabolic problem of the type $u_{t}-\sum_{i=1}^{n}\left(\partial / \partial x_{i}\right) a_{i}(x, t, u, \nabla u)=f(x, t),(x, t) \in Q$; $u(x, t)=0,(x, t) \in \partial \Omega \times(0, T) ; u(x, 0)=0, x \in \Omega$, where $p>1, \Omega$ is a nonempty, bounded, and open subset of $\mathbb{R}^{N}, a_{i}:$ $\Omega \times(0, T) \times \mathbb{R} \times \mathbb{R}^{N} \rightarrow \mathbb{R}(i=1,2, \ldots, n)$ satisfies certain growth conditions, and $f \in L^{p^{\prime}}(Q), Q=\Omega \times(0, T)$, and $p^{\prime}$ is the conjugate exponent of $p$.
\end{abstract}

\section{Introduction-Preliminaries}

In what follows, $X$ is a real reflexive separable locally uniformly convex Banach space with locally uniformly convex dual space $X^{*}$. The norm of the space $X$, and any other normed spaces herein, will be denoted by $\|\cdot\|$. For $x \in X$ and $x^{*} \in X^{*}$, the pairing $\left\langle x^{*}, x\right\rangle$ denotes the value $x^{*}(x)$. Let $X$ and $Y$ be real Banach spaces. For a multivalued mapping $T: X \rightarrow 2^{Y}$, we define the domain $D(T)$ of $T$ by $D(T)=\{x \in$ $X: T x \neq \emptyset\}$ and the range $R(T)$ of $T$ by $R(T)=\cup_{x \in D(T)} T x$. We also denote the graph of $T$ by $G(T)=\{(x, T x): x \in D(T)\}$. A mapping $T: X \supset D(T) \rightarrow Y$ is "demicontinuous" if it is continuous from the strong topology of $D(T)$ to the weak topology of $Y$. A multivalued mapping $T: X \supset D(T) \rightarrow 2^{Y}$ is "bounded" if it maps bounded subsets of $D(T)$ to bounded subsets of $Y$. It is "compact" if it is strongly continuous and maps bounded subsets of $D(T)$ to relatively compact subset of $Y$. It is "finitely continuous" if it is upper semicontinuous from each finite dimensional subspace $F$ of $X$ to the weak topology of $Y$. It is "quasibounded" if for every $M>0$ there exists $K(M)>0$ such that $\left[x, w^{*}\right] \in G(T)$ with $\|x\| \leq M$ and $\left\langle w^{*}, x\right\rangle \leq M\|x\|$ imply $\left\|w^{*}\right\| \leq K(M)$. It is "strongly quasibounded" if for every $M>0$ there exists $K(M)>0$ such that $\left[x, w^{*}\right] \in G(T)$ with $\|x\| \leq M$ and $\left\langle w^{*}, x\right\rangle \leq M$ imply $\left\|w^{*}\right\| \leq K(M)$. In what follows, a mapping will be called "continuous" if it is strongly continuous.

Let $\psi:[0, \infty) \rightarrow[0, \infty)$ be continuous strictly increasing function such that $\psi(0)=0$ and $\psi(t) \rightarrow \infty$ as $t \rightarrow \infty$. The duality mapping corresponding to $\psi$ denoted by $J_{\psi}: X \rightarrow 2^{X^{*}}$ is defined by

$$
\begin{aligned}
& J_{\psi}(x) \\
& \quad=\left\{x^{*} \in X^{*}:\left\langle x^{*}, x\right\rangle=\left\|x^{*}\right\|\|x\|,\left\|x^{*}\right\|=\psi(\|x\|)\right\} .
\end{aligned}
$$

It is well-known that, for each $x \in X$, the Hahn-Banach Theorem implies $J_{\psi}(x) \neq \emptyset$. Since $X$ and $X^{*}$ are locally 
uniformly convex, $J_{\psi}$ is single-valued, bounded monotone of type $\left(S_{+}\right)$and bicontinuous. If $\psi(t)=t$ for $t \geq 0$, then $J_{\psi}$ is denoted by $J$ and is called the normalized duality mapping.

An operator $T: X \supset D(T) \rightarrow 2^{X^{*}}$ is said to be "monotone" if, for every $x \in D(T), y \in D(T)$, and every $u^{*} \in T x, v^{*} \in T y$, we have $\left\langle u^{*}-v^{*}, x-y\right\rangle \geq 0$. A monotone mapping $T: X \supset D(T) \rightarrow 2^{X^{*}}$ is "maximal monotone" if $R(T+\lambda J)=X^{*}$ for every $\lambda>0$; that is, $T$ is maximal monotone if and only if $T$ is monotone and $\left\langle u^{*}-u_{0}^{*}, x-x_{0}\right\rangle \geq 0$ for every $\left(x, u^{*}\right) \in G(T)$ implies $x_{0} \in D(T)$ and $u_{0}^{*} \in T x_{0}$. If $T$ is maximal monotone, the operator $T_{t}: X \rightarrow X^{*}, t \in(0, \infty)$, defined by $T_{t} x=$ $\left(T^{-1}+t J^{-1}\right)^{-1} x$, is bounded, continuous, maximal monotone and such that $T_{t} x \rightarrow T^{(0)} x$ as $t \rightarrow 0^{+}$, for every $x \in D(T)$, where $\left\|T^{(0)} x\right\|=\inf \left\{\left\|y^{*}\right\|: y^{*} \in T x\right\}$. The "resolvent" $J_{t}: X \rightarrow D(T)$, defined by $J_{t} x=x-t J^{-1}\left(T_{t} x\right)$, is continuous and $T_{t} x \in T\left(J_{t} x\right)$ for every $x \in X$. Moreover, $\lim _{t \rightarrow 0} J_{t} x=x$ for all $x \in \overline{\operatorname{coD} D(T)}$, where $\operatorname{coD}(T)$ is the convex hull of the set $D(T)$. An operator $A: X \supseteq D(A) \rightarrow 2^{X^{*}}$ is called "coercive" if either $D(A)$ is bounded or there exists a function $\psi:[0, \infty) \rightarrow(-\infty, \infty)$ such that $\psi(t) \rightarrow \infty$ as $t \rightarrow \infty$ and $\left\langle y^{*}, x\right\rangle \geq \psi(\|x\|)\|x\|$ for all $x \in D(A)$ and $y^{*} \in A x$. For an operator $A: X \supseteq D(A) \rightarrow 2^{X^{*}}$ and $x \in D(A)$, we denote $|A x|=\inf \left\{\left\|v^{*}\right\|: v^{*} \in A x\right\}$. It is called weakly coercive if either $D(A)$ is bounded or $|A x| \rightarrow \infty$ as $\|x\| \rightarrow \infty$.

The following definitions are used throughout the paper. In arbitrary Banach space $X$, Browder and Hess [1] introduced the definitions of pseudomonotone and generalized pseudomonotone operators. The original definition for single-valued pseudomonotone, generalized pseudomonotone, and operators of type $(M)$ with domain all of $X$, is due to Brézis [2].

Definition 1. An operator $S: X \supseteq D(S) \rightarrow X^{*}$ is called

(i) "generalized pseudomonotone" if, for each sequence $\left\{x_{n}\right\}$ in $D(S)$ with $x_{n} \rightarrow x_{0}$ and $S x_{n} \rightarrow v_{0}^{*}$ as $n \rightarrow \infty$ such that $\lim \sup _{n \rightarrow \infty}\left\langle S x_{n}, x_{n}-x_{0}\right\rangle \leq 0$, then $x_{0} \epsilon$ $D(S), S x_{0}=v_{0}^{*}$, and $\left\langle S x_{n}, x_{n}\right\rangle \rightarrow\left\langle S x_{0}, x_{0}\right\rangle$ as $n \rightarrow$ $\infty$.

(ii) "type $(M)$ " if, for each sequence $\left\{x_{n}\right\}$ in $D(S)$ with $x_{n} \rightarrow x_{0}$ in $X$ and $S x_{n} \rightarrow v_{0}^{*}$ as $n \rightarrow \infty$ such that $\lim \sup _{n \rightarrow \infty}\left\langle S x_{n}, x_{n}-x_{0}\right\rangle \leq 0$, then $x_{0} \in D(S)$ and $S x_{0}=v_{0}^{*}$.

(iii) " $\alpha$-expansive" if there exists $\alpha>0$ such that $\| v^{*}$ $u^{*}\|\geq \alpha\| x-y \|$ for all $x \in D(S), y \in D(S), v^{*} \in S x$, and $u^{*} \in S y$. It is called expansive if $\alpha=1$.

We notice here that the definition of single-valued expansive mapping is due to Nirenberg [3]. In order to enlarge the class of single-valued operators, the multivalued version is introduced in (iii) of Definition 1. It is not hard to notice that every uniformly monotone operator is expansive. Furthermore, in a Hilbert space $X=H$, if $T: H \supseteq D(T) \rightarrow$ $2^{H}$ is monotone, we see that, for each $\lambda>0, T+\lambda I$ is multivalued expansive with domain $D(T)$.
The following definition gives a larger class of operators of monotone type, which can be found in Kartsatos and Skrypnik [4].

Definition 2. Let $T: X \supseteq D(T) \rightarrow 2^{X^{*}}$ be maximal monotone and $A: X \supseteq D(A) \rightarrow X^{*}$. Let $L \subseteq D(M) \cap D(A)$ be a linear subspace of $X$. Then $A$ is said to be

(i) "quasibounded with respect to $T$ " if, for each $M>0$, there exists $K(M)>0$ such that

$$
\begin{aligned}
\left\langle A u+u^{*}, u\right\rangle & \leq M, \\
\|u\| & \leq M,
\end{aligned}
$$

where $u \in L$ and $u^{*} \in T u$, then $\|A u\| \leq K(M)$,

(ii) "generalized $\left(S_{+}\right)$with respect to $T$ " if, for each $\left\{u_{n}\right\}$ in $L$ with $u_{n}^{*} \in T u_{n}, u_{n} \rightarrow u_{0}$ in $X$ and $A u_{n} \rightarrow h_{0}^{*}$ in $X^{*}$ as $n \rightarrow \infty$ such that

$$
\begin{array}{r}
\limsup _{n \rightarrow \infty}\left\langle A u_{n}, u_{n}-u_{0}\right\rangle \leq 0, \\
\left\langle u_{n}^{*}+A u_{n}, u_{n}\right\rangle \leq 0
\end{array}
$$

for all $n$, then $u_{n} \rightarrow u_{0} \in D(A)$ and $A u_{0}=h_{0}^{*}$,

(iii) "generalized pseudomonotone with respect to $T$ " if, for each $\left\{u_{n}\right\}$ in $L$ with $u_{n}^{*} \in T u_{n}, u_{n} \rightarrow u_{0}$ in $X$ and $A u_{n} \rightarrow h_{0}^{*}$ in $X^{*}$ as $n \rightarrow \infty$ such that

$$
\begin{array}{r}
\limsup _{n \rightarrow \infty}\left\langle A u_{n}, u_{n}-u_{0}\right\rangle \leq 0, \\
\left\langle u_{n}^{*}+A u_{n}, u_{n}\right\rangle \leq 0
\end{array}
$$

for all $n$, then $u_{0} \in D(A), A u_{0}=h_{0}^{*}$, and $\left\langle S u_{n}, u_{n}\right\rangle \rightarrow$ $\left\langle S u_{0}, u_{0}\right\rangle$ as $n \rightarrow \infty$,

(iv) "of type $(M)$ with respect to $T$ " if, for each $\left\{u_{n}\right\}$ in $L$ with $u_{n}^{*} \in T u_{n}, u_{n} \rightarrow u_{0}$ in $X$ and $A u_{n} \rightarrow h_{0}^{*}$ in $X^{*}$ as $n \rightarrow \infty$ such that

$$
\begin{array}{r}
\limsup _{n \rightarrow \infty}\left\langle A u_{n}, u_{n}-u_{0}\right\rangle \leq 0, \\
\left\langle u_{n}^{*}+A u_{n}, u_{n}\right\rangle \leq 0
\end{array}
$$

for all $n$, then $u_{0} \in D(A)$ and $A u_{0}=h_{0}^{*}$.

By Definition 2, it is not difficult to see that $0 \in D(T)$ and $A$ is quasibounded implying that $A$ is quasibounded with respect to $T$. Furthermore, it follows that the class of generalized $\left(S_{+}\right)$operators with respect to $T$ includes the class of operators of type $\left(S_{+}\right)$.

For basic definitions and further properties of mappings of monotone type, the reader is referred to Barbu [5], Brèzis et al. [6], Brèzis [2], Browder and Hess [1], Pascali and Sburlan [7], Browder [8], and Zeidler [9]. For results concerning perturbations of maximal monotone operators by bounded and everywhere defined pseudomonotone type operators, the reader is referred to Browder and Hess [1], Brèzis [2], Browder [10], Brèzis and Nirenberg [11], Kenmochi [1214], Guan et al. [15], Le [16], Guan and Kartsatos [15, 17], 
and Kartsatos and Skrypnik [4] and the references therein. For recent degree theory and applications for solvability of operator inclusions involving bounded pseudomonotone perturbations of maximal monotone operators under general coercivity and Leray-Schauder type boundary conditions, we cite the paper due to Asfaw and Kartsatos [18]. Existence results concerning noncoercive operators of the type $T+S$, where $T: X \supseteq D(T) \rightarrow 2^{X^{*}}$ is maximal monotone and $S: X \rightarrow 2^{X^{*}}$ is bounded pseudomonotone, can be found in the paper due to Asfaw [19]. For applications of the theory of perturbed monotone type operators to variational and hemivariational inequality problems, the reader is referred to the papers due to Carl and Le [20], Carl et al. [21], Carl [22], and Carl and Motreanu [23] and the references therein. For a separable reflexive Banach space $X$ and a nonempty, closed, and convex subset $K$ of $X$, Asfaw and Kartsatos [24] gave existence results for locally defined operators of the type $T+S$, where $T: X \supseteq D(T) \rightarrow 2^{X^{*}}$ is maximal monotone and $S: K \rightarrow X^{*}$ is demicontinuous and generalized pseudomonotone under coercivity condition on $S$.

The main contribution of the paper is to obtain surjectivity results for noncoercive and not everywhere defined operators of the type

(i) $T+S$, where $S: X \supseteq D(S) \rightarrow X^{*}$ is quasibounded demicontinuous generalized pseudomonotone such that

(a) there exists a real reflexive separable Banach space $W \subseteq D(S)$, dense and continuously embedded in $X$;

(b) there exists $d \geq 0$ such that $\left\langle v^{*}+S x, x\right\rangle \geq$ $-d\|x\|^{2}$ for all $x \in D(T) \cap D(S)$ and $v^{*} \in T x$;

(c) there exist $\alpha>d$ and $\mu \geq 0$ such that $\left\|v^{*}+S x\right\| \geq$ $\alpha\|x\|-\mu$ for all $x \in D(T) \cap D(S)$ and $v^{*} \in T x$,

(ii) $L+S$, where $S: X \supseteq D(S) \rightarrow X^{*}$ is quasibounded demicontinuous of type $(M)$ with $D(L) \subseteq D(S)$ such that (b) and (c) of (i) are satisfied.

In Section 2, we proved surjectivity results for $T+S$ and $L+S$ satisfying conditions (i) and (ii), respectively. In Theorem 6 , we provide a surjectivity result for operators of the type $T+S$, where $T$ and $S$ satisfy condition (i). Theorem 6 is new and improves the existing surjectivity results for an operator $S$, which is single-valued, everywhere defined, bounded, and coercive pseudomonotone. In particular, for a single-valued pseudomonotone operator $S$, Theorem 6 improves the surjectivity results due to Browder and Hess [1], Kenmochi [12-14], Le [16], Guan and Kartsatos [17], Asfaw and Kartsatos [18], and Asfaw [19, 25] because the results in these references require $S$ to be everywhere defined, bounded, and coercive while Theorem 6 used $S$ to be densely defined, quasibounded, and noncoercive. Moreover, Browder (cf. Zeidler [9, Theorem 32. A, pages 866-872]) gave the main theorem for perturbations of maximal monotone operator by a single-valued, bounded, demicontinuous, and coercive operator $S$ with $D(S)=C$, a nonempty, closed, and convex subset of $X$. In view of this, Theorem 6 gives an analogous result, where $D(S)$ is dense in $X$, possibly, neither closed nor convex, and $S$ is weakly coercive. It is also known, due to Browder and Hess [1], that every pseudomonotone operator $S$ from $X$ into $X^{*}$ with $D(S)=X$ is generalized pseudomonotone. It is also true that $S$ is demicontinuous provided that it is bounded, single-valued, and everywhere defined. Consequently, the arguments used in the proof of Theorem 6 give analogous conclusion if $S: X=D(S) \rightarrow$ $X^{*}$ is bounded pseudomonotone and $T$ and $S$ satisfy the given hypotheses. As a consequence of Corollary 7, a partial positive answer for Nirenberg's problem on surjectivity of densely defined demicontinuous generalized pseudomonotone expansive mapping is provided. In addition, Theorem 8 provides surjectivity result for operators of the type $T+S$, where $T$ and $S$ satisfy condition (ii). As a result of Theorem 8 , a new characterization of linear maximal monotone operator is proved when the space $X$ is separable. It is well known due to Brézis (cf. Zeidler [9, Theorem 32. L, pages 897-899]) that a linear monotone operator $L$ is maximal monotone if and only if $L$ is closed and densely defined and the adjoint operator $L^{*}$ is monotone. An interesting result in the present paper is that a linear monotone operator $L$ is maximal monotone if and only if $L$ is closed and densely defined, provided that $X$ is separable. This result weakens the monotonicity condition on $L^{*}$ used by Brézis (cf. Zeidler [9, Theorem 32. L, pages 897-899]). To the best of the author's knowledge, Theorem 8 is a new result and Corollary 9 improves the well-known result of Brézis. In Section 3, we demonstrate the applicability of the results by proving existence of weak solution in $L^{p}\left(0, T ; W_{0}^{1, p}(\Omega)\right)$ of a nonlinear parabolic problem, where $p>1$ and $\Omega$ is a nonempty, bounded, and open subset of $\mathbb{R}^{N}$.

The following important lemma is due to Brèzis et al. [6].

Lemma 3. Let $B$ be a maximal monotone set in $X \times X^{*}$. If $\left(u_{n}, u_{n}^{*}\right) \in B$ such that $u_{n} \rightarrow u, u_{n}^{*} \rightarrow u^{*}$ as $n \rightarrow \infty$, and either

$$
\limsup _{n, m \rightarrow \infty}\left\langle u_{n}^{*}-u_{m}^{*}, u_{n}-u_{m}\right\rangle \leq 0
$$

or

$$
\limsup _{n \rightarrow \infty}\left\langle u_{n}^{*}-u^{*}, u_{n}-u\right\rangle \leq 0,
$$

then $\left(u, u^{*}\right) \in B$ and $\left\langle u_{n}^{*}, u_{n}\right\rangle \rightarrow\left\langle u^{*}, u\right\rangle$ as $n \rightarrow \infty$.

Browder and Ton [26] gave the following important embedding result.

Lemma 4. Let $X$ be a separable reflexive Banach space. Then there exists a real separable Hilbert space $H$ and a compact injection $Q: H \rightarrow X$ such that $\overline{Q(H)}=X$.

In this paper, we use the following fixed point result for compact operators, originally due to Leray and Schauder, which may be found in the book of Granas and Dugundji [27, Theorem 5.2, page 123].

Lemma 5. Let $C$ be a convex subset of a normed linear space $X$ and let $U$ be nonempty relatively open in $C$ with $0 \in U$. Then each compact map $F: \bar{U} \rightarrow C$ satisfies that either 
(i) F has a fixed point in $U$

or

(ii) there exist $x \in \partial_{C} U$ and $\lambda \in(0,1)$ such that $x=\lambda F(x)$, where $\partial_{C} U$ is the boundary of $U$ with respect to the subspace topology on $C$.

\section{Main Results}

In this section, we prove the following new surjectivity result for maximal monotone perturbation of densely defined noncoercive generalized pseudomonotone operator in separable reflexive Banach spaces.

Theorem 6. Let $T: X \supseteq D(T) \rightarrow 2^{X^{*}}$ be maximal monotone with $0 \in T(0)$ and $S: X \supseteq D(S) \rightarrow X^{*}$ quasibounded demicontinuous generalized pseudomonotone. Suppose $W \subseteq D(S)$ is a real reflexive separable Banach space dense and continuously embedded in X. Assume, further, that there exist $\mu \geq 0, d \geq 0$, and $\alpha>d$ satisfying

$$
\langle S x, x\rangle \geq-d\|x\|^{2}
$$

for all $x \in D(S)$ and either

(i)

$$
\left\|v^{*}+S x\right\| \geq \alpha\|x\|-\mu \quad \forall x \in D(T) \cap D(S), v^{*} \in T x
$$

or

(ii) there exists $\phi:[0, \infty) \rightarrow(-\infty, \infty)$ such that $\phi(t) \rightarrow$ $\infty$ as $t \rightarrow \infty$ and

$$
\left\|v^{*}+S x\right\| \geq \phi(\|x\|)\|x\|
$$

$$
\forall x \in D(T) \cap D(S), v^{*} \in T x .
$$

Then $T+S$ is surjective.

Proof. Let $\lambda>0$ be fixed temporarily and $T_{\lambda}$ the Yosida approximant of $T$. For each $\varepsilon>0$, by using the inner product condition on $S$ and monotonicity of $T_{\lambda}\left(T_{\lambda}(0)=0\right.$ for all $\lambda>0$ ), we see that

$$
\begin{aligned}
& \left\langle T_{\lambda} x+S x+\delta\|x\| J x-f^{*}, x\right\rangle \\
& \geq \delta\|x\|^{3}-d\|x\|^{2}-\left\|f^{*}\right\|\|x\| \\
& =\|x\|^{3}\left[\delta-\frac{d}{\|x\|}-\frac{\left\|f^{*}\right\|}{\|x\|^{3}}\right]>0
\end{aligned}
$$

for all $x \in D(S) \cap \partial B_{R_{\delta}}(0)$ for some $R_{\delta}>0$. Let $G_{\delta}=B_{R_{\delta}}(0)$. Let $H$ be a real separable Hilbert space and $Q: H \rightarrow W$ a compact injection such that $Q(H)$ is dense in $W$ guaranteed by Lemma 4 . Let $j: W \rightarrow X$ be the natural injection and let $Q^{*}: W^{*} \rightarrow H^{*}$ and $j^{*}: X^{*} \rightarrow W^{*}$ be adjoint of $Q$ and $j$, respectively. It follows that $\psi=j Q: H \rightarrow X$ is a compact operator. Let $U=Q^{-1}\left(G_{\delta} \cap W\right)$. First we show that $G_{\delta} \cap W$ is open in $W$; that is, $W \backslash\left(G_{\delta} \cap W\right)=W \cap\left(X \backslash G_{\delta}\right)$ is closed in
$W$. To this end, let $\left\{x_{n}\right\}$ be a sequence in $W \cap\left(X \backslash G_{\delta}\right)$ such that $x_{n} \rightarrow x_{0}$ in $W$ as $n \rightarrow \infty$. Since $W$ is continuously embedded in $X$, we get $x_{n} \rightarrow x_{0}$ in $X$ as $n \rightarrow \infty$. Since $X \backslash G_{\delta}$ is closed in $X$, it follows that $x_{0} \in X \backslash G_{\delta}$; that is, $x_{0} \in W \cap\left(X \backslash G_{\delta}\right)$. This shows that $W \cap\left(X \backslash G_{\delta}\right)$ is closed in $W$; that is, $G_{\delta} \cap W$ is open in $W$. The continuity of $Q$ implies that $U$ is open in $H$. Since $W$ is continuously embedded in $X$, it follows that

$$
{\overline{G_{\delta} \cap W}}^{W} \subseteq{\overline{G_{\delta} \cap W}}^{X} \subseteq \overline{G_{\delta}}
$$

where the closures are taken with respect to the spaces $W$ and $X$, respectively. Since ${\overline{G_{\delta} \cap W}}^{W} \subseteq W$, we obtain that

$$
\begin{aligned}
\left(G_{\delta} \cap W\right) \cup \partial_{W}\left(G_{\delta} \cap W\right)={\overline{G_{\delta} \cap W}}^{W} \subseteq \overline{G_{\delta}} \cap W \\
\quad=\left(G_{\delta} \cap W\right) \cup\left(\partial G_{\delta} \cap W\right) .
\end{aligned}
$$

Since the sets $G_{\delta} \cap W$ and $\partial_{W}\left(G_{\delta} \cap W\right)$ are disjoint, we conclude that

$$
\partial_{W}\left(G_{\delta} \cap W\right) \subseteq \partial G_{\delta} \cap W
$$

For each $\lambda>0$, let $T_{\lambda}$ be the Yosida approximant of $T$. Let $J_{1} x=\|x\| J x, x \in X$. It is known that, for each $\delta>0, T_{\lambda}+\delta J_{1}$ is bounded, continuous, monotone, and of type $\left(S_{+}\right)$. Let $\psi=$ $j Q$ and $C_{\varepsilon}^{\lambda, \delta}: \bar{U} \rightarrow H$ be given by

$$
C_{\varepsilon}^{\lambda, \delta}(v)=-\varepsilon^{-1}\left(\psi^{*}\left(\left(T_{\lambda}+S+\delta J_{1}\right) \psi(v)-f^{*}\right)\right),
$$

$$
v \in \bar{U} \text {. }
$$

Since $\psi$ is continuous, it follows that

$$
\bar{U}=\overline{\psi^{-1}\left(G_{\delta} \cap W\right)} \subseteq \psi^{-1}\left({\overline{G_{\delta} \cap W}}^{W}\right) \subseteq H
$$

is closed subset of $H$. We show that $C_{\varepsilon}^{\lambda, \delta}$ is a compact operator. To this end, let $x_{n} \in \bar{U}$ such that $x_{n} \rightarrow x_{0}$ as $n \rightarrow \infty$. Since $Q$ is continuous from $H$ into $X$, we have $Q x_{n} \rightarrow Q x_{0}$ as $n \rightarrow \infty$. Since $x_{n} \in \bar{U}$, the sequence $\left\{Q x_{n}\right\}$ lies in $W$. Since $x_{n} \in H$ for all $n$ and $x_{0} \in \bar{U}$, it follows that $Q x_{n} \in W$ and $\mathrm{Q} x_{0} \in W$ for all $n$. Since $S$ and $T_{\lambda}$ are demicontinuous, it follows that $\left(T_{\lambda}+S\right) Q x_{n} \rightarrow\left(T_{\lambda}+S\right) Q x_{0}$ as $n \rightarrow \infty$. By the density of $W$ in $X$, it is known that $j^{*}$ is defined from $W^{*}$ into $H$. As a result, for each $w \in W$, we see that

$$
\begin{aligned}
\left\langle j^{*}\right. & \left.\left(T_{\lambda}+S+\delta J_{1}\right) Q x_{n}-j^{*}\left(T_{\lambda}+S+\delta J_{1}\right) Q x_{0}, w\right\rangle \\
= & \left\langle\left(T_{\lambda}+S+\delta J_{1}\right) Q x_{n}, w\right\rangle \\
& -\left\langle\left(T_{\lambda}+S+\delta J_{1}\right) Q x_{0}, w\right\rangle
\end{aligned}
$$

for all $n$. However, the right side expression goes to 0 as $n \rightarrow$ $\infty$; that is, for each $w \in W$, it follows that

$$
\begin{aligned}
& \left\langle j^{*}\left(T_{\lambda}+S+\delta J_{1}\right) Q x_{n}-j^{*}\left(T_{\lambda}+S+\delta J_{1}\right) Q x_{0}, w\right\rangle \\
& \quad \longrightarrow 0 \quad \text { as } n \longrightarrow \infty .
\end{aligned}
$$


On the other hand, by the density of $W$ in $X$, for each $x \in X$, we get

$$
\begin{aligned}
& \left\langle j^{*}\left(T_{\lambda}+S+\delta J_{1}\right) Q x_{n}-j^{*}\left(T_{\lambda}+S+\delta J_{1}\right) Q x_{0}, x\right\rangle \\
& \quad \longrightarrow 0 \quad \text { as } n \longrightarrow \infty ;
\end{aligned}
$$

that is, $j^{*}\left(T_{\lambda}+S+\delta J_{1}\right) Q x_{n} \rightarrow j^{*}\left(T_{\lambda}+S+\delta J_{1}\right) Q x_{0}$ as $n \rightarrow \infty$. Since $Q^{*}$ is compact linear, which is completely continuous and $(j Q)^{*}=Q^{*} j^{*}$, we arrive at $\psi^{*}\left(T_{\lambda}+S+\delta J_{1}\right) Q x_{n} \rightarrow$ $\psi^{*}\left(T_{\lambda}+S+\delta J_{1}\right) Q x_{0}$ as $n \rightarrow \infty$. This shows that the mapping $C_{\varepsilon}^{\delta, \lambda}$ is continuous. Following similar argument as above, it is not difficult to show that $C_{\varepsilon}^{\delta, \lambda}$ maps any bounded subset of $\bar{U}$ into relatively compact subset of $H$. As a result, we conclude that $C_{\varepsilon}^{\delta, \lambda}$ is a compact operator. Fix $\varepsilon>0$. In order to use Lemma 5, it is enough to show that (i) of Lemma 5 does not hold; that is, for all $\mu \in(0,1)$ and $x \in \partial_{H} U$, we have $x \neq$ $\mu C_{\varepsilon}^{\delta, \lambda}(x)$. Suppose this is false; that is, there exist $x_{0} \in \partial_{H} U$ and $\mu_{0} \in(0,1)$ such that $x_{0}=\mu_{0} C_{\varepsilon}^{\delta, \lambda}\left(x_{0}\right)$. This yields

$$
\varepsilon x_{0}+\mu_{0} \psi^{*}\left(\left(T_{\lambda}+S+\delta J_{1}\right) \psi x_{0}-f^{*}\right)=0 \text {. }
$$

We notice here that the continuity of $Q$, property of $Q^{-1}$, and definition of boundary of an open set imply that

$$
\begin{aligned}
\partial_{H} U & =\partial_{H} Q^{-1}\left(G_{\delta} \cap W\right) \subseteq Q^{-1}\left(\partial_{W}\left(G_{\delta} \cap W\right)\right) \\
& \subseteq Q^{-1}\left(\partial G_{\delta} \cap W\right)
\end{aligned}
$$

holds. Since $x_{0} \in \partial_{H} U$, it follows that $Q x_{0} \in \partial G \cap W$. By (11) and (20), we get

$$
\begin{aligned}
\frac{\varepsilon}{\mu_{0}}\left\|x_{0}\right\|^{2} & =-\left\langle\psi^{*}\left(\left(T_{\lambda}+S+\delta J_{1}\right) Q x_{0}-f^{*}\right), x_{0}\right\rangle \\
& =-\left\langle\left(T_{\lambda}+S+\delta J_{1}\right) Q x_{0}-f^{*}, Q x_{0}\right\rangle \leq 0,
\end{aligned}
$$

which implies $x_{0}=0$. But this is impossible because $0 \in G_{\delta} \cap$ $W$. Therefore, by applying Lemma 5 , for each $\varepsilon>0, \lambda>0$, and $\delta>0$, we conclude that the compact operator $C_{\varepsilon}^{\delta, \lambda}$ has a fixed point $x_{\varepsilon} \in U$; that is,

$$
\varepsilon x_{\varepsilon}+\psi^{*}\left(\left(T_{\lambda}+S+\delta J_{1}\right) \psi x_{\varepsilon}-f^{*}\right)=0 .
$$

Therefore, for each $\varepsilon_{n} \downarrow 0^{+}$, there exists $x_{n} \in \bar{U}$ such that

$$
\varepsilon_{n} x_{n}+\psi^{*}\left(\left(T_{\lambda}+S+\delta J_{1}\right) \psi x_{n}-f^{*}\right)=0
$$

for all $n$. Since $G_{\delta}$ is bounded, the sequence $\left\{\psi x_{n}\right\}$ is bounded. Since $T_{\lambda}$ and $J_{1}$ are bounded, it follows that the sequence $\left\{\left(T_{\lambda}+\delta J_{1}\right) \psi x_{n}\right\}$ is bounded. Since $W=\overline{Q(H)}^{W}$ and $W$ is continuously embedded, we see that $W=\overline{Q(H)}^{W} \subseteq \overline{\mathrm{Q}(H)}^{X}$, where the closures are with respect to the norms in $W$ and $X$, respectively. As a result, the density of $W$ in $X$ implies that
$X=\overline{\mathrm{Q}(H)}^{\mathrm{X}}$. By using (11), the monotonicity of $T_{\lambda}$ and $J_{1}$, and property of $\psi^{*}$, we obtain that

$$
\begin{aligned}
\langle S \psi & \left.x_{n}, \psi x_{n}\right\rangle \\
= & -\varepsilon_{n}\left\|x_{n}\right\|^{2} \\
& -\left\langle T_{\lambda} \psi x_{n}+\delta J_{1} \psi x_{n}-\left(T_{\lambda}(0)+\delta J_{1}(0)\right), \psi x_{n}\right\rangle \\
& +\left\langle T_{\lambda}(0)+\delta J_{1}(0)+f^{*}, \psi x_{n}\right\rangle \\
\leq & \left\|T_{\lambda}(0)+\delta J_{1}(0)+f^{*}\right\|\left\|\psi x_{n}\right\| \\
\leq & \left(|T(0)|+\left\|f^{*}\right\|\right)\left\|\psi x_{n}\right\|
\end{aligned}
$$

for all $n$. Since $Q x_{n} \in W$, it follows that $\psi x_{n}=j Q x_{n}=Q x_{n}$ for all $n$. Consequently, we obtain that

$$
\left\langle S Q x_{n}, Q x_{n}\right\rangle \leq\left(|T(0)|+\left\|f^{*}\right\|\right)\left\|Q x_{n}\right\|
$$

for all $n$. Since $\left\{Q x_{n}\right\}$ is bounded and $S$ is quasibounded, we conclude that $\left\{S Q x_{n}\right\}$ is bounded. Consequently, by using (24), it is not difficult to see that $\left\{\varepsilon_{n}\left\|x_{n}\right\|^{2}\right\}$ is bounded. If the sequence $\left\{x_{n}\right\}$ is bounded, then $\varepsilon_{n} x_{n} \rightarrow 0$ as $n \rightarrow \infty$. Otherwise, by using the boundedness of $\left\{\varepsilon_{n}\left\|x_{n}\right\|^{2}\right\}$, we assume without loss of generality that $\varepsilon_{n} x_{n} \rightarrow 0$ as $n \rightarrow \infty$, $\mathrm{Q} x_{n} \rightarrow x_{0}$, and $S Q x_{n} \rightarrow v_{0}^{*}$ as $n \rightarrow \infty$. Since $\overline{Q(H)}^{X}=X$, by choosing a sequence $\left\{z_{m}=Q y_{m}\right\}$ such that $z_{m} \rightarrow x_{0}$ as $m \rightarrow \infty$ and using (24) together with the monotonicity of $T_{\lambda}+\delta J_{1}$, we get

$$
\begin{aligned}
& \left\langle S Q x_{n}, Q x_{n}\right\rangle=\left\langle S Q x_{n}, Q y_{m}-Q y_{m}+Q x_{n}\right\rangle \\
& =\left\langle S Q x_{n}, Q y_{m}\right\rangle+\left\langle S Q x_{n}, Q x_{n}-Q y_{m}\right\rangle \\
& =\left\langle S Q x_{n}, Q y_{m}\right\rangle+\left\langle f^{*}, Q x_{n}-Q y_{m}\right\rangle \\
& -\left\langle\left(T_{\lambda}+\delta J_{1}\right) Q x_{n}-\left(T_{\lambda}+\delta J_{1}\right) Q y_{m}, Q x_{n}-Q y_{m}\right\rangle \\
& -\left\langle\left(T_{\lambda}+\delta J_{1}\right) Q y_{m}, Q x_{n}-Q y_{m}\right\rangle \\
& -\varepsilon_{n}\left\langle x_{n}, x_{n}-y_{m}\right\rangle \leq\left\langle S Q x_{n}, Q y_{m}\right\rangle \\
& -\left\langle\left(T_{\lambda}+\delta J_{1}\right) Q y_{m}, Q x_{n}-Q y_{m}\right\rangle+\varepsilon_{n}\left\langle x_{n}, y_{m}\right\rangle \\
& +\left\langle f^{*}, Q x_{n}-Q y_{m}\right\rangle
\end{aligned}
$$

for all $n$ and $m$. Fixing $m$ and letting $n \rightarrow \infty$ in (27), we obtain that

$$
\begin{aligned}
\limsup _{n \rightarrow \infty} & \left\langle S Q x_{n}, Q x_{n}\right\rangle \\
\leq & \left\langle v_{0}^{*}, Q y_{m}\right\rangle \\
& -\left\langle\left(T_{\lambda}+\delta J_{1}\right) Q y_{m}-f^{*}, x_{0}-Q y_{m}\right\rangle .
\end{aligned}
$$

Since $T_{\lambda}+\delta J_{1}$ is demicontinuous, letting $m \rightarrow \infty$, we arrive at

$$
\limsup _{n \rightarrow \infty}\left\langle S Q x_{n}, Q x_{n}\right\rangle \leq\left\langle v_{0}^{*}, x_{0}\right\rangle
$$

that is,

$$
\limsup _{n \rightarrow \infty}\left\langle S Q x_{n}, Q x_{n}-x_{0}\right\rangle \leq 0 \text {. }
$$


Since $S$ is generalized pseudomonotone, we conclude that $x_{0} \in D(S), S x_{0}=v_{0}^{*}$, and $\left\langle S Q x_{n}, Q x_{n}\right\rangle \rightarrow\left\langle v_{0}^{*}, x_{0}\right\rangle$ as $n \rightarrow \infty$. For any $y \in Q(H)$, applying the monotonicity of $T_{\lambda}+\delta J_{1}$, we arrive at

$$
\begin{aligned}
& \left\langle T_{\lambda} y+\delta J_{1} y-f^{*}, y-x_{0}\right\rangle=\lim _{n \rightarrow \infty}\left\langle T_{\lambda} y+\delta J_{1} y\right. \\
& \left.-f^{*}, y-Q x_{n}\right\rangle \geq \liminf _{n \rightarrow \infty}\left[\left\langleT_{\lambda} y+\delta J_{1} y\right.\right. \\
& \left.\left.-\left(T_{\lambda} Q x_{n}+\delta J_{1} Q x_{n}\right), y-Q x_{n}\right\rangle\right] \\
& +\liminf _{n \rightarrow \infty}\left[\left\langle T_{\lambda} Q x_{n}+\delta J_{1} Q x_{n}-f^{*}, y-Q x_{n}\right\rangle\right] \\
& \geq \liminf _{n \rightarrow \infty}\left\langle T_{\lambda} Q x_{n}+\delta J_{1} Q x_{n}-f^{*}, y-Q x_{n}\right\rangle .
\end{aligned}
$$

Moreover, from (24), we obtain that

$$
\begin{aligned}
& \left\langle\left(T_{\lambda}+\delta J_{1}\right) Q x_{n}-f^{*}, y-Q x_{n}\right\rangle \\
& \quad=-\varepsilon_{n}\left\langle x_{n}, Q^{-1} y-x_{n}\right\rangle-\left\langle S Q x_{n}, y-Q x_{n}\right\rangle \\
& \quad=\varepsilon_{n}\left\|x_{n}\right\|^{2}-\varepsilon_{n}\left\langle x_{n}, Q^{-1} y\right\rangle-\left\langle S Q x_{n}, y-Q x_{n}\right\rangle \\
& \quad \geq-\varepsilon_{n}\left\langle x_{n}, Q^{-1} y\right\rangle-\left\langle S Q x_{n}, y-Q x_{n}\right\rangle
\end{aligned}
$$

for all $n$. As a result, we arrive at

$$
\begin{aligned}
& \liminf _{n \rightarrow \infty}\left\langle\left(T_{\lambda}+\delta J_{1}\right) Q x_{n}-f^{*}, y-Q x_{n}\right\rangle \\
& \geq-\left\langle S x_{0}, y-x_{0}\right\rangle .
\end{aligned}
$$

From (31) and (33), we obtain

$$
\left\langle\left(T_{\lambda}+\delta J_{1}\right) y+S x_{0}-f^{*}, y-x_{0}\right\rangle \geq 0
$$

for all $y \in Q(H)$. By the density of $Q(H)$ in $X$ and the continuity of $T_{\lambda}+\delta J_{1}$, we conclude that

$$
\left\langle T_{\lambda} y+\delta J_{1} y+S x_{0}-f^{*}, y-x_{0}\right\rangle \geq 0
$$

for all $y \in X$. Since, for any $y \in X, x_{t}=t x_{0}+(1-t) y \in X$ for all $t \in[0,1)$, using $x_{t}$ in place of $y$, we obtain that

$$
\left\langle\left(T_{\lambda}+\delta J_{1}\right) x_{t}+S x_{0}-f^{*},(1-t)\left(y-x_{0}\right)\right\rangle \geq 0
$$

for all $t \in[0,1)$; that is,

$$
\left\langle\left(T_{\lambda}+\delta J_{1}\right) x_{t}+S x_{0}-f^{*}, y-x_{0}\right\rangle \geq 0
$$

for all $t \in[0,1)$. Since $T_{\lambda}+\delta J_{1}$ is continuous and $x_{t} \rightarrow x_{0}$ as $t \rightarrow 1^{-}$, we have $T_{\lambda} x_{t}+\delta J_{1} x_{t} \rightarrow T_{\lambda} x_{0}+\delta J_{1} x_{0}$ as $t \rightarrow 1^{-}$. Letting $t \rightarrow 1^{-}$, we arrive at

$$
\left\langle\left(T_{\lambda}+\delta J_{1}\right) x_{0}+S x_{0}-f^{*}, y-x_{0}\right\rangle \geq 0
$$

for all $y \in X$. Since $y \in X$ is arbitrary, setting $y+x_{0}$ in place of $y$ yields

$$
\left\langle\left(T_{\lambda}+\delta J_{1}\right) x_{0}+S x_{0}-f^{*}, y\right\rangle \geq 0
$$

for all $y \in X$. Therefore, for each $\lambda>0$ (by fixing $\delta>0$ temporarily), we see that there exists $x_{\lambda} \in D(S) \cap \bar{G}_{\delta}$ such that $T_{\lambda} x_{\lambda}+\delta J_{1} x_{\lambda}+S x_{\lambda}=f^{*}$. Thus, for each $\lambda_{n} \downarrow 0^{+}$, there exists $y_{n} \in D(S) \cap \bar{G}_{\delta}$ such that

$$
T_{\lambda_{n}} y_{n}+\delta J_{1} y_{n}+S y_{n}-f^{*}=0
$$

for all $n$. Since $\bar{G}_{\delta}$ and $J_{1}$ are bounded, it follows that $\left\{y_{n}\right\}$ and $\left\{J_{1} y_{n}\right\}$ are bounded. Since $S$ is quasibounded, it is not hard to see that $\left\{S y_{n}\right\}$ is bounded, which implies the boundedness of $\left\{T_{\lambda_{n}} y_{n}\right\}$. Assume without loss of generality that $y_{n} \rightarrow y_{0}$, $S y_{n} \rightarrow v_{0}^{*}$ and $T_{\lambda_{n}} y_{n} \rightarrow u_{0}^{*}$ as $n \rightarrow \infty$. Since $S+\delta J_{1}$ is generalized pseudomonotone with domain $D(S)$, it follows that

$$
\liminf _{n \rightarrow \infty}\left\langle S y_{n}+\delta J_{1} y_{n}, y_{n}-y_{0}\right\rangle \geq 0
$$

Consequently, from (40), we arrive at

$$
\limsup _{n \rightarrow \infty}\left\langle T_{\lambda_{n}} y_{n}, y_{n}-y_{0}\right\rangle \leq 0 .
$$

Let $J_{\lambda_{n}}$ be the Yosida resolvent of $T$. It is well known that $J_{\lambda_{n}} y_{n} \in D(T), J_{\lambda_{n}} y_{n}=x_{n}-\lambda_{n} J^{-1}\left(T_{\lambda_{n}} y_{n}\right)$, and $T_{\lambda_{n}} y_{n} \in$ $T\left(J_{\lambda_{n}} y_{n}\right)$ for all $n$. Since $y_{n} \rightarrow y_{0}$ and $\left\{T_{\lambda_{n}} y_{n}\right\}$ is bounded, it follows that $J_{\lambda_{n}} y_{n} \rightarrow y_{0}$ as $n \rightarrow \infty$. Thus, we have

$$
\begin{aligned}
\left\langle T_{\lambda_{n}} y_{n}, J_{\lambda_{n}} y_{n}-y_{0}\right\rangle= & \left\langle T_{\lambda_{n}} y_{n}, J_{\lambda_{n}} y_{n}-y_{n}\right\rangle \\
& +\left\langle T_{\lambda_{n}} y_{n}, y_{n}-y_{0}\right\rangle \\
= & -\left\langle T_{\lambda_{n}} y_{n}, \lambda_{n} J^{-1}\left(T_{\lambda_{n}} y_{n}\right)\right\rangle \\
& +\left\langle T_{\lambda_{n}} y_{n}, y_{n}-y_{0}\right\rangle \\
= & -t_{n}\left\|T_{\lambda_{n}} y_{n}\right\|^{2} \\
& +\left\langle T_{\lambda_{n}} y_{n}, y_{n}-y_{0}\right\rangle \\
\leq & \left\langle T_{\lambda_{n}} y_{n}, y_{n}-y_{0}\right\rangle
\end{aligned}
$$

for all $n$. Consequently, we have

$$
\begin{aligned}
& \lim _{n \rightarrow \infty}\left\langle T_{\lambda_{n}} y_{n}, J_{\lambda_{n}} y_{n}-y_{0}\right\rangle \leq \lim _{n \rightarrow \infty}\left\langle T_{\lambda_{n}} y_{n}, y_{n}-y_{0}\right\rangle \\
& \quad \leq 0 .
\end{aligned}
$$

By the maximality of $T$, applying Lemma 3, we obtain $x_{0} \in$ $D(T), v_{0}^{*} \in T x_{0}$, and $\left\langle T_{\lambda_{n}} y_{n}, J_{\lambda_{n}} y_{n}\right\rangle \rightarrow\left\langle v_{0}^{*}, x_{0}\right\rangle$ as $n \rightarrow \infty$, which implies

$$
\limsup _{n \rightarrow \infty}\left\langle S y_{n}, y_{n}-y_{0}\right\rangle \leq 0
$$

The generalized pseudomonotonicity of $S$ implies $y_{0} \in D(S)$ and $S y_{0}=h_{0}^{*}$. As a result, letting $n \rightarrow \infty$ in (40), we conclude that $v_{0}^{*}+S y_{0}+\delta J_{1} y_{0}=f^{*}$. This implies that, for each $\delta_{n} \downarrow 0^{+}$, there exist $z_{n} \in D(T) \cap D(S)$ and $v_{n}^{*} \in T z_{n}$ such that

$$
v_{n}^{*}+S z_{n}+\delta_{n} J_{1} z_{n}=f^{*}
$$

for all $n$. Next we will show that $\left\{z_{n}\right\}$ is bounded. Assume without loss of generality that $\left\|z_{n}\right\| \rightarrow \infty$ as $n \rightarrow \infty$. By 
the inner product condition on $S$ and monotonicity of $T$ with $0 \in T(0)$, we get

$$
\delta_{n}\left\|z_{n}\right\|^{3} \leq d\left\|z_{n}\right\|^{2}+\left\|f^{*}\right\|\left\|z_{n}\right\|
$$

for all $n$; that is, dividing this inequality by $\left\|z_{n}\right\|$ for all large $n$, we get $\delta_{n}\left\|z_{n}\right\|^{2} \leq d\left\|z_{n}\right\|+\left\|f^{*}\right\|$ for all large $n$. By using condition $(i)$ and (46), we get that

$$
\begin{aligned}
-\mu+\alpha\left\|z_{n}\right\| & \leq\left\|v_{n}^{*}+S z_{n}\right\| \leq \delta_{n}\left\|z_{n}\right\|^{2}+\left\|f^{*}\right\| \\
& \leq d\left\|z_{n}\right\|+2\left\|f^{*}\right\|
\end{aligned}
$$

for all $n$. This gives $(\alpha-d)\left\|z_{n}\right\| \leq 2\left\|f^{*}\right\|+\mu$ for all $n$. Consequently, the boundedness of $\left\{z_{n}\right\}$ follows. Since $S$ is quasibounded and $0 \in D(T)$, it is not hard to see that $\left\{S z_{n}\right\}$ is bounded. Consequently, the boundedness of $\left\{v_{n}^{*}\right\}$ follows. Assuming that $z_{n}^{*} \rightarrow z_{0}^{*}, v_{n}^{*} \rightarrow v_{0}^{*}$, and $S z_{n} \rightarrow h_{0}^{*}$ as $n \rightarrow \infty$ and using the arguments used in the first half of the proof of Theorem 6, we conclude that $z_{0} \in D(T) \cap D(S), v_{0}^{*} \in T z_{0}$, $S z_{0}=h_{0}^{*}$, and $v_{0}^{*}+S z_{0}=f^{*}$; that is, for each $f^{*} \in X^{*}$, the inclusion problem $T u+S u \ni f^{*}$ is solvable in $D(T) \cap$ $D(S)$. Since $f^{*} \in X^{*}$ is arbitrary, we obtain the surjectivity of $T+S$. The proof using condition (ii) can be completed following similar arguments. The details are omitted here. This completes the proof.

It is worth mentioning that Theorem 6 is a new result because the perturbed operator $T+S$ is noncoercive and $S$ is densely defined such that $D(S)$ contains a dense real separable reflexive Banach space. Under the conditions on $T+$ $S$, the result was unknown earlier even for coercive operator $T+S$. The analog of Theorem 6 for single multivalued, finitely continuous, coercive, and quasibounded generalized pseudomonotone operator $S$ such that $D(S)$ contains a dense linear subspace is due to Browder and Hess [1]. If $S$ is quasimonotone with weakly closed graph or graph of $T$ is weakly closed and $S$ is monotone of type $(M)$, the arguments used in the proof of Theorem 6 can be easily carried out to conclude the surjectivity of $T+S$. The reader is referred to Gupta [28] for a result for $T+S$, where graph of $T$ is assumed to be weakly closed and $S: X \supseteq D(S) \rightarrow 2^{X^{*}}$ is quasibounded, finitely continuous coercive operator of type $(M)$ such that $D(S)$ contains a dense linear subspace. Theorem 6 improves and gives unifications of the existing surjectivity results due to Le [16], Asfaw and Kartsatos [18, 24], Asfaw [19], and Kenmochi [12-14] for maximal monotone perturbations of coercive bounded pseudomonotone operators with domain, all of $X$. In addition, it can be easily seen that the proof of Theorem 6 can go through if the quasiboundedness of $S$ is omitted and $T$ is assumed to be strongly quasibounded with $0 \in T(0)$. Another observation is that the condition $\left\langle v^{*}+S x, x\right\rangle \geq-d\|x\|^{2}$ for all $x \in D(T) \cap D(S)$ and $v^{*} \in T x$ can be replaced by a stronger condition $\left\langle v^{*}+S x, x\right\rangle \geq-d\|x\|$ for all $x \in D(T) \cap D(S)$ and $v^{*} \in T x$, and the weak coercivity condition (i) can be relaxed to satisfy $|T x+S x| \rightarrow \infty$ as $\|x\| \rightarrow \infty$. On the other hand, one can easily see that weak coercivity condition on $T+S$ is automatically satisfied if $T+S$ is $\alpha$-expansive. Consequently, the following corollary is immediate.
Corollary 7. Let $T: X \supseteq D(T) \rightarrow 2^{X^{*}}$ be strongly quasibounded maximal monotone with $0 \in T(0)$ and let $S: X \supseteq D(S) \rightarrow X^{*}$ be demicontinuous generalized pseudomonotone. Suppose $W \subseteq D(S)$ is a real reflexive separable Banach space dense and continuously embedded in $X$. Assume, further, that $T+S$ is $\alpha$-expansive mapping and there exists $d \geq 0$ such that $\alpha>d$ and $\langle S x, x\rangle \geq-d\|x\|^{2}$ for all $x \in D(S)$. Then $T+S$ is surjective.

Proof. Since $0 \in T 0$ and $T$ is monotone, by the condition on $S$, it follows that $\left\langle v^{*}+S x, x\right\rangle \geq-d\|x\|^{2}$ for all $x \in D(T) \cap D(S)$. Furthermore, by the expansiveness of $T+S$, for some $u_{0} \in$ $D(T) \cap D(S)$ and $v_{0}^{*} \in T u_{0}$, we arrive at

$$
\begin{aligned}
\left\|v^{*}+S x\right\| & \geq\left\|v^{*}+S x-\left(v_{0}^{*}+S u_{0}\right)\right\|-\left\|v_{0}^{*}+S u_{0}\right\| \\
& \geq \alpha\left\|x-u_{0}\right\|-\left\|v_{0}^{*}+S u_{0}\right\| \\
& \geq \alpha\|x\|-\alpha\left\|u_{0}\right\|-\left\|v_{0}^{*}+S u_{0}\right\| \\
& =\alpha\|x\|-A_{0},
\end{aligned}
$$

where $A_{0}=\alpha\left\|u_{0}\right\|+\left\|v_{0}^{*}+S u_{0}\right\|$, for all $x \in D(T) \cap D(S)$ and $v^{*} \in T x$. This shows that $T+S$ satisfies conditions of Theorem 6. By applying similar arguments as in the last part of the proof of Theorem 6 and using the strong quasiboundedness of $T$ instead of quasiboundedness of $S$, we conclude that $T+S$ is surjective. The details are omitted here.

It is worth noticing here that Corollary 7 gives a partial positive answer for Nirenberg's problem on the surjectivity of expansive mapping in a real separable reflexive Banach space. More precisely, Corollary 7 gives surjectivity of densely defined demicontinuous generalized pseudomonotone expansive mapping. To the best of the authors knowledge, this result was unknown. For related surjectivity results for continuous expansive mappings in a real Hilbert space, we cite the papers by Kartsatos [29] and Xiang [30]. For range result for single continuous quasimonotone expansive mapping defined from arbitrary reflexive Banach space into its dual space $X^{*}$, the reader is referred to the paper due to Asfaw [25].

The content of the following theorem addresses the solvability of operator equations involving operators of the type $L+S$, where $L: X \supseteq D(L) \rightarrow X^{*}$ is linear, densely defined, monotone, and closed, and $S: X \supseteq D(S) \rightarrow X^{*}$ is quasibounded demicontinuous of type $(M)$ such that $D(L)$ lies in $D(S)$.

Theorem 8. Let $L: X \supseteq D(L) \rightarrow X^{*}$ be closed, densely defined, and linear monotone, and let $S: X \supseteq D(S) \rightarrow X^{*}$ be quasibounded demicontinuous of type $(M)$ with respect to $L$ such that $D(L)$ lies in $D(S)$. Assume, further, that there exist $\mu \geq 0$ and $\alpha>d \geq 0$ such that

$$
\langle L x+S x, x\rangle \geq-d\|x\|^{2}
$$

for all $x \in D(L)$ and either

(i)

$$
\|L x+S x\| \geq \alpha\|x\|-\mu \quad \forall x \in D(L)
$$


or

(ii) there exists $\phi:[0, \infty) \rightarrow(-\infty, \infty)$ such that $\phi(t) \rightarrow$ $\infty$ ast $\rightarrow \infty$ and

$$
\|L x+S x\| \geq \phi(\|x\|)\|x\| \quad \forall x \in D(L) .
$$

Then $L+S$ is surjective.

Proof. Fix $f^{*} \in X^{*}$. Let $Y=D(L)$ and let $\|\cdot\|_{Y}$ be the graph norm on $Y$ given by

$$
\|x\|_{Y}=\|x\|_{X}+\|L x\|_{X^{*}}, \quad x \in Y .
$$

It is well-known that $Y$ equipped with the graph norm becomes a real reflexive separable Banach space. By Lemma 4, let $H$ be a Hilbert space and let $Q: H \rightarrow Y$ be a compact injection such that $Q(H)$ is dense in $Y$. Let $j: Y \rightarrow X$ be the natural embedding of $Y$ into $X$ and let $j^{*}: X^{*} \rightarrow Y^{*}$ be its adjoint. It follows that $\psi=j Q$ is a compact injection from $H$ into $X$. By using the graph norm on $Y$, it follows that $Y$ is dense and continuously embedded in $X$. Moreover, by the inner product condition on $L+S$, for each $\delta>0$, there exists $R_{\delta}>0$ such that

$$
\begin{aligned}
\left\langle L x+S x+\delta J_{1} x, x\right\rangle & \geq \delta\|x\|^{3}-d\|x\|^{2} \\
& =\|x\|^{3}\left[\delta-\frac{d}{\|x\|}\right]>0
\end{aligned}
$$

for all $x \in D(L) \cap D(S) \cap \partial B_{R_{\delta}}(0)$. Let $G_{\delta}=B_{R_{\delta}}(0)$. By using the arguments used in the first half of the proof of Theorem 6 , we see that $G_{\delta} \cap Y$ is open in $Y$ and $\partial_{Y}\left(G_{\delta} \cap Y\right) \subseteq \partial G_{\delta} \cap Y$. Let $U=\psi^{-1}\left(G_{\delta} \cap Y\right)$. Since $j: Y \rightarrow X$ and $Q: H \rightarrow Y$ are continuous, it follows that $U$ is open in $H$. Since the operator $j^{*} L j: Y \rightarrow Y^{*}$ is linear and monotone, it is continuous. By the arguments used in the proof of Theorem 6, using $Y$ in place of $X$ and the closed convex subset $\bar{U}$ of $H$, it follows that the mapping $C_{\varepsilon}: \bar{U} \rightarrow H$ defined by

$$
C_{\varepsilon}(v)=-\varepsilon^{-1}\left(\psi^{*}\left(L+S+\delta J_{1}\right) J v-f^{*}\right), \quad v \in \bar{U}
$$

is compact. In addition, we see that

$$
\begin{aligned}
\partial_{H} U & =\partial_{H} \psi^{-1}\left(G_{\delta} \cap Y\right) \subseteq \psi^{-1}\left(\partial_{Y}\left(G_{\delta} \cap Y\right)\right) \\
& \subseteq \psi^{-1}\left(\partial G_{\delta} \cap Y\right) .
\end{aligned}
$$

Following the argument as in the proof of Theorem 6, it is not difficult to see that $x \neq \lambda C_{\varepsilon}(x)$ for all $x \in \partial_{H} U$ and all $\lambda \epsilon$ $(0,1)$. Consequently, by Lemma 5 , we obtain that, for each $\varepsilon>$ $0, C_{\varepsilon}$ has a fixed point in $U$. Therefore, for each $\varepsilon_{n} \downarrow 0^{+}$, there exists $x_{n} \in U$ such that

$$
\varepsilon_{n} x_{n}+\psi^{*}\left(L+S+\delta J_{1}\right) \psi x_{n}=\psi^{*} f^{*}
$$

for all $n$; that is,

$$
\begin{gathered}
\left\langle\varepsilon_{n} x_{n}, x\right\rangle+\left\langle Q^{*}\left(j^{*}\left(L+S+\delta J_{1}\right) j Q x_{n}\right), x\right\rangle \\
=\left\langle Q^{*} j^{*}\left(f^{*}\right), x\right\rangle \quad \forall x \in H .
\end{gathered}
$$

Since $j: Y \rightarrow X$ and $Q: H \rightarrow Y$, by the definition of $Q^{*}$ and $j^{*}$, we see that

$$
\begin{aligned}
\left\langle\varepsilon_{n} x_{n}, x\right\rangle+\left\langle\left(L+S+\delta J_{1}\right) Q x_{n}, Q x\right\rangle=\left\langle f^{*}, Q x\right\rangle & \\
& \forall x \in H .
\end{aligned}
$$

Since $\psi x_{n} \in G_{\delta} \cap Y$ and $G_{\delta}$ is bounded in $X$, it follows that the sequence $\left\{j x_{n}\right\}=\left\{Q x_{n}\right\}$ is bounded in $X$. From (57), using the monotonicity $L(L(0)=0)$, boundedness of $\left\{Q x_{n}\right\}$, and quasiboundedness of $S$, we get the boundedness of the sequence $\left\{S Q x_{n}\right\}$. This gives

$$
\begin{aligned}
\varepsilon_{n}\left\|x_{n}\right\|^{2} & =-\left\langle Q^{*}\left(L+S+\delta J_{1}\right) Q x_{n}-Q^{*} f^{*}, x_{n}\right\rangle \\
& \leq-\left\langle S Q x_{n}+\delta J_{1} Q x_{n}-f^{*}, Q x_{n}\right\rangle \\
& \leq\left\|S Q x_{n}+\delta J_{1} Q x_{n}-f^{*}\right\|\left\|Q x_{n}\right\| \quad \forall n .
\end{aligned}
$$

As a result, we get the boundedness of $\left\{\varepsilon_{n}\left\|x_{n}\right\|^{2}\right\}$. If $\left\{x_{n}\right\}$ is bounded, then $\varepsilon_{n} x_{n} \rightarrow 0$ as $n \rightarrow \infty$. If $\left\{x_{n}\right\}$ is unbounded, by passing into a subsequence, we see that

$$
\varepsilon_{n}\left\|x_{n}\right\|=\frac{\varepsilon_{n}\left\|x_{n}\right\|^{2}}{\left\|x_{n}\right\|} \longrightarrow 0 \text { as } n \longrightarrow \infty \text {. }
$$

In all cases, we assume without loss of generality that $\varepsilon_{n} x_{n} \rightarrow$ 0 as $n \rightarrow \infty$. As a result, we get

$$
\begin{aligned}
\left\langle L Q x_{n}, Q x\right\rangle= & -\left\langle\varepsilon_{n} x_{n}, x\right\rangle-\left\langle S Q x_{n}+\delta J_{1} Q x_{n}, Q x\right\rangle \\
& +\left\langle f^{*}, Q x\right\rangle \\
\leq & \left\|\varepsilon_{n} x_{n}+S Q x_{n}+\delta J_{1} Q x_{n}+f^{*}\right\|\|Q x\| \\
\leq & \mu\|Q x\|,
\end{aligned}
$$

where $\mu$ is an upper bound for the sequence $\left\{\| \varepsilon_{n} x_{n}+S Q x_{n}+\right.$ $\left.\delta J_{1} Q x_{n}+f^{*} \|\right\}$. Since $Q(H)$ is dense in $Y$, for each $y \in Y$, there exists a sequence $\left\{y_{m}\right\}$ in $H$ such that $Q y_{m} \rightarrow y$ as $n \rightarrow \infty$. This gives

$$
\begin{aligned}
\left|\left\langle L Q x_{n}, y\right\rangle\right| & =\lim _{m \rightarrow \infty}\left|\left\langle L Q x_{n}, Q y_{m}\right\rangle\right| \leq \lim _{m \rightarrow \infty} \mu\left\|Q y_{m}\right\| \\
& =\mu\|y\| .
\end{aligned}
$$

By similar argument, the density of $Y$ in $X$ implies that

$$
\left|\left\langle L Q x_{n}, x\right\rangle\right| \leq \mu\|x\| \quad \forall x \in X .
$$

By using the uniform boundedness principle, we conclude that $\left\{L Q x_{n}\right\}$ is bounded. Assume without loss of generality that $Q x_{n} \rightarrow x_{0}$ in $X, S Q x_{n} \rightarrow v_{0}^{*}$, and $L Q x_{n} \rightarrow h^{*}$ in $X^{*}$ as $n \rightarrow \infty$. Since $L$ is closed linear, it follows that $x_{0} \in Y$ and $h^{*}=L x_{0}$. By following the arguments used in the first half of the proof of Theorem 6 along with (57), we get

$$
\limsup _{n \rightarrow \infty}\left\langle S Q x_{n}-f^{*}, Q x_{n}-x_{0}\right\rangle \leq 0 \text {. }
$$

On the other hand, from (58), by using $x_{n} \in H$ in place of $x$, we see that

$$
\left\langle L Q x_{n}+S Q x_{n}-f^{*}, Q x_{n}\right\rangle \leq 0 \quad \forall n .
$$


Since $S$ is of type $(M)$ with respect to $L$, it follows that $S-f^{*}$ is also of type $(M)$ with respect to $L$, which yields $x_{0} \in D(S)$ and $v_{0}^{*}=S x_{0}$. Finally, letting $n \rightarrow \infty$ in (57), we get $\psi^{*}(L+$ $\left.S+\delta J_{1}\right) x_{0}=\psi^{*} f^{*}$; that is, $Q^{*} j^{*}\left(L+S+\delta J_{1}\right) x_{0}=Q^{*} j^{*} f^{*}$. Since $Q(H)$ and $Y$ are dense in $Y$ and $X$, respectively, it follows that $j^{*}$ and $Q^{*}$ are one to one. Therefore, we arrive at $L x_{0}+$ $S x_{0}+\delta J_{1} x_{0}=f^{*}$. Consequently, for each $\delta_{n} \downarrow 0^{+}$, there exists $y_{n} \in D(L)$ such that

$$
L y_{n}+S y_{n}+\delta_{n} J_{1} y_{n}=f^{*} \quad \forall n \text {. }
$$

Since $L$ is closed and $S$ is of type $(M)$, by weak coercivity condition on $L+S$, the same arguments used in the second half of the proof of Theorem 6 can be carried over to conclude the solvability of the equation $L x+S x \ni f^{*}$ in $D(L)$. Since $f^{*} \in X^{*}$ is arbitrary, we conclude that $L+S$ is surjective. The details are omitted here.

The following corollary gives a characterization of linear maximal monotone operator in separable reflexive Banach space.

Corollary 9. Let $X$ be a real separable reflexive Banach space and let $L: X \supseteq D(L) \rightarrow X^{*}$ be linear operator. Then the following two statements are equivalent:

(i) L is maximal monotone,

(ii) L is monotone, densely defined, and closed.

Proof. The proof of (i) implies (ii) follows by the well-known result due to Brézis (cf. Zeidler [9, Theorem 32. L, page 897]). Next we prove (ii) implies (i). Let $\lambda>0$. It is sufficient to show that $R(L+\lambda J)=X^{*}$. To this end, we will use Theorem 8 . Since $L$ is linear and monotone, that is, $\langle L x, x\rangle \geq 0$ for all $x \in D(L)$, and $J$ is monotone, it follows that $\langle L x+\lambda J x, x\rangle \geq \lambda\|x\|^{2}$ for all $x \in D(L)$. Therefore, for each $\lambda>0$, it follows that

$$
\|L x+\lambda J x\| \geq \lambda\|x\| \quad \forall x \in D(L) .
$$

By using $J$ in place of $S$ in Theorem 8 , we conclude that $R(L+$ $\lambda J)=X^{*}$ for any $\lambda>0$. Thus, $L$ is maximal monotone.

It is worth noticing that Brézis proved (i) in arbitrary reflexive Banach space provided that $L^{*}$ is monotone and (ii) holds. As a result, Corollary 9 is an improvement of the result of Brézis when $X$ is separable. It is important to mention that Gupta [28] gave surjectivity result for graph weakly closed maximal monotone perturbations of quasibounded, finitely continuous multivalued coercive operator $S$ of type $(M)$ such that $D(S)$ contains a dense linear subspace of $X$. However, the result in Theorem 8 is for noncoercive operator $S$ along with weak coercivity of $L+S$. It is also important to mention here that the results in Theorems 6 and 8 are new even in the case where the operator $S$ is coercive but not everywhere defined. In conclusion, Theorems 6 and 8 gave improvements over the existing theory for maximal monotone perturbations of coercive and everywhere defined operators of pseudomonotone type.

\section{Example and Discussion}

In this section, we demonstrate the existence of weak solution in $X=L^{p}\left(0, T ; W_{0}^{1, p}(\Omega)\right)$ for the parabolic problem of the type

$$
\begin{aligned}
\frac{\partial u}{\partial t}-\sum_{i=1}^{N} \frac{\partial}{\partial x_{i}} a_{i}(x, t, u, \nabla u) & =f(x, t) \quad(x, t) \in Q \\
u(x, t) & =0 \quad(x, t) \in \partial \Omega \times(0, T) \\
u(x, 0) & =0 \quad x \in \Omega,
\end{aligned}
$$

where $p>1, p^{\prime}$ is conjugate exponent of $p, Q=\Omega \times(0, T)$, $\Omega$ is a nonempty, bounded, and open subset of $\mathbb{R}^{N}$, and $f \in$ $L^{p^{\prime}}(Q)$ such that the following conditions are satisfied:

$\left(A_{1}\right) a_{i}(x, t, s, \xi)(i=1,2, \ldots N)$ satisfies the Carathéodory conditions; that is, for each $(s, \xi) \in \mathbb{R} \times \mathbb{R}^{N}$, the function $(x, t) \mapsto a_{i}(x, t, s, \xi)$ is measurable and, for almost all $(x, t) \in \Omega \times(0, T)$, the function $(s, \xi) \mapsto$ $a_{i}(x, t, s, \xi)$ is continuous.

$\left(A_{2}\right)$ there exists positive constants $\mu_{1}$ and $\mu_{2}$ such that

$$
\begin{aligned}
& \sum_{i=1}^{N}\left(a_{i}(x, t, s, \xi)-a_{i}(x, t, s, \eta)\right)\left(\xi_{i}-\eta_{i}\right) \\
& \quad \geq \mu_{1}|\xi-\eta|^{p}, \\
& \left|a_{i}(x, t, s, \xi)\right| \leq \mu_{2}\left[|s|^{p_{0}}+|\xi|^{p-1}\right]+g(x, t)
\end{aligned}
$$

for all $(x, t, s, \xi) \in \Omega \times(0, T) \times \mathbb{R} \times \mathbb{R}^{N}$, where $\xi=$ $\left(\xi_{i}\right) \in \mathbb{R}^{N}$ and $\eta=\left(\eta_{i}\right) \in \mathbb{R}^{N}, g \in L^{p^{\prime}}(Q), p_{0}<$ $p-1+2(p-1) / N$, and $1<p<N$.

Let $X=L^{p}(0, T ; V), V=W_{0}^{1, p}(\Omega)$, and $L: X \supseteq D(L) \rightarrow X^{*}$ be defined by $L u=u^{\prime}$, where $u^{\prime}$ is understood in the sense of distributions; that is,

$$
\int_{0}^{T} u^{\prime}(t) \psi(t) d t=-\int_{0}^{T} u(t) \psi^{\prime}(t) d t
$$

$$
\psi \in C_{0}^{\infty}(0, T)
$$

where $D(L)=\left\{u \in X: u^{\prime} \in X^{*}, u(0)=0\right\}$. We notice that

$$
\langle L u, \phi\rangle=\int_{0}^{T}\left\langle u^{\prime}(t), \phi(t)\right\rangle_{V} d t
$$

$$
u \in D(L), \phi \in X \text {. }
$$

Let $A: X \supseteq D(A) \rightarrow X^{*}$ be defined by

$$
\begin{aligned}
\langle A u, \phi\rangle=\sum_{i=1}^{n} \int_{Q} a_{i}(x, t, u, \nabla u) \frac{\partial \phi(x, t)}{\partial x_{i}} d x d t, & \\
\phi \in X, u \in D(A), &
\end{aligned}
$$

where

$$
D(A)=\left\{u \in X: u \in L^{\bar{p}}(Q)\right\}, \quad \bar{p}=\frac{p_{0} p}{p-1} .
$$


It is known from Kartsatos and Skrypnik [4] that $A$ is quasibounded demicontinuous generalized $\left(S_{+}\right)$with respect to $L$ such that $D(A)$ contains $D(L)$. Moreover, it is wellknown that $L$ is linear, closed, and densely defined maximal monotone. The operator $A$ is densely defined, that is, not everywhere defined, and coercive. Since $p_{0}<p-1$ in $\left(A_{2}\right)$, operator $A$ may be unbounded. Therefore, by Theorem 8 using $A$ in place of $S$, for each $f \in L^{p^{\prime}}(Q)$, we conclude that the equation $A u+L u=f^{*}$ is solvable in $D(L)$, where $f^{*}: X \rightarrow \mathbb{R}$ is given by $\left\langle f^{*}, \phi\right\rangle=\int_{Q} f(x, t) \phi(x, t) d x d t$. Therefore, the parabolic problem (69) admits at least one weak solution in $D(L)$.

Since $\Omega$ is bounded and $g \in L^{p^{\prime}}(Q)$, it is well known that $A$ is bounded, continuous, everywhere defined, and coercive provided that $p_{0}=p-1$ in $\left(A_{2}\right)$. More precisely, these conditions on $A$ are satisfied if condition $\left(A_{2}\right)$ is replaced by

$\left(A_{3}\right)$ : there exists positive constants $\mu_{1}$ and $\mu_{2}$ such that

$$
\begin{aligned}
& \sum_{i=1}^{N}\left(a_{i}(x, t, s, \xi)-a_{i}(x, t, s, \eta)\right)\left(\xi_{i}-\eta_{i}\right) \geq \mu_{1}|\xi-\eta|^{p} \\
& \left|a_{i}(x, t, s, \xi)\right| \leq \mu_{2}\left[|s|^{p-1}+|\xi|^{p-1}\right]+g(x, t) \\
& \quad \text { for all }(x, t, s, \xi) \in \Omega \times(0, T) \times \mathbb{R} \times \mathbb{R}^{N}, \text { where } \xi=\left(\xi_{i}\right) \in \\
& \mathbb{R}^{N} \text { and } \eta=\left(\eta_{i}\right) \in \mathbb{R}^{N}, g \in L^{p^{\prime}}(Q), \text { and } 1<p<N .
\end{aligned}
$$

Abstract existence results concerning nonlinear parabolic problems of the type in (69) under conditions $\left(A_{1}\right)$ and $\left(A_{3}\right)$ have been intensively studied by many researchers. For some of the basic and relevant references, the reader is referred to the papers by Browder and Hess [1], Brézis [2], Le [16], Kenmochi [12-14], Guan and Kartsatos [17], Asfaw and Kartsatos [18], Asfaw [19, 25], and the references therein. For further examples and applications of perturbed everywhere defined pseudomonotone type operators to inclusion, variational inequality, and evolution problems, the reader is referred to the papers of Landes and Mustonen [31], Kobayashi and Ôtani [32], and Mustonen [33] and books of Kinderlehrer and Stampacchia [34], Browder [8], and Naniewicz and Panagiotopoulos [35] and the references therein. The method of sub-supersolution is employed in the papers by Carl and Le [20], Carl et al. [21], Carl [22], Carl and Motreanu [23], and Le $[36,37]$ to study existence and properties of solution(s) for evolution inclusion problems of the type

$$
u \in X: u^{\prime}+A(u) \ni f^{*} \quad \text { in } X^{*}, u(0)=u_{0} \text {, }
$$

where $X=L^{p}\left(0, T ; W_{0}^{1, p}(\Omega)\right), p>1, \Omega$ is a nonempty, bounded, and open subset of $\mathbb{R}^{N}$, and $A$ is noncoercive but still everywhere defined operator of pseudomonotone type. For further relevant information about sub-supersolution arguments concerning evolution type problems, the reader is referred to the recent book on nonsmooth analysis due to Carl et al. [38] and the references therein. Finally, it is important to indicate the readers that the results in this paper can be conveniently applied to address nonlinear parabolic problems of type (76) as well as elliptic problems of the type $u \in Y:-\Delta_{p} u+B(u) \ni g^{*}, g^{*} \in Y^{*}$, where $Y=W_{0}^{1, p}(\Omega)$ and $A$ and $B$ are possibly noncoercive and densely defined and satisfy conditions of either Theorem 6 or Theorem 8 .

\section{Conflict of Interests}

The author declares that there is no conflict of interests regarding the publication of this paper.

\section{Acknowledgments}

The author would like thank the editor and anonymous referee for forwarding important suggestions. Furthermore, the author would like to thank the Virginia Tech's Open Access Sub-vention fund program for covering the full article processing fee.

\section{References}

[1] F. E. Browder and P. Hess, "Nonlinear mappings of monotone type in Banach spaces," Journal of Functional Analysis, vol. 11, no. 3, pp. 251-294, 1972.

[2] H. Brèzis, "Équations et inéquations non linéaires dans les espaces vectoriels en dualité," Annales de l'Institut Fourier, vol. 18, no. 1, pp. 115-175, 1968.

[3] L. Nirenberg, Topics in Nonlinear Functional Analysis, Lecture Notes, Courant Institute of Mathematical Sciences, New York University, New York, NY, USA, 1974.

[4] A. G. Kartsatos and I. V. Skrypnik, "Topological degree theories for densely defined mappings involving operators of type $(S+)$," Advances in Differential Equations, vol. 4, no. 3, pp. 413-456, 1999.

[5] V. Barbu, Nonlinear Semigroups and Differential Equations in Banach Spaces, Noordhoff International Publishing, Leyden, The Netherlands, 1975.

[6] H. Brèzis, M. G. Crandall, and A. Pazy, "Perturbations of nonlinear maximal monotone sets in banach space," Communications on Pure and Applied Mathematics, vol. 23, no. 1, pp. 123-144, 1970.

[7] D. Pascali and S. Sburlan, Nonlinear Mappings of Monotone Type, Sijthoff and Noordhoof, Bucharest, Romania, 1978.

[8] F. E. Browder, "Nonlinear operators and nonlinear equations of evolution in Banach spaces," Proceedings of Symposia in Pure Mathematics, vol. 1, pp. 1-308, 1976.

[9] E. Zeidler, Nonlinear Functional Analysis and Its Applications, Springer, New York, NY, USA, 1990.

[10] F. E. Browder, "Pseudo-monotone operators and nonlinear elliptic boundary value problems on unbounded domains," Proceedings of the National Academy of Sciences of the United States of America, vol. 74, no. 7, pp. 2659-2661, 1977.

[11] H. Brèzis and L. Nirenberg, "Characterizations of the ranges of some nonlinear operators and applications to boundary value problems," Annali della Scuola Normale Superiore di Pisa, vol. 5, pp. 225-326, 1978.

[12] N. Kenmochi, "Nonlinear operators of monotone type in reflexive Banach spaces, and nonlinear perturbations," Hiroshima Mathematical Journal, vol. 4, no. 1, pp. 229-263, 1974.

[13] N. Kenmochi, "Pseudomonotone operators and nonlinear ellpitic boundary value problems," Journal of the Mathematical Society of Japan, vol. 27, pp. 121-149, 1975. 
[14] N. Kenmochi, "Monotonicity and compactness methods for nonlinear variational inequalities," in Handbook of Differential Equations, IV, pp. 203-298, Elsevier/North-Holland, Amsterdam, The Netherlands, 2007.

[15] Z. Guan, A. G. Kartsatos, and I. V. Skrypnik, "Ranges of densely defined generalized pseudomonotone perturbations of maximal monotone operators," Journal of Differential Equations, vol. 188, no. 1, pp. 332-351, 2003.

[16] V. K. Le, "A range and existence theorem for pseudomonotone perturbations of maximal monotone operators," Proceedings of the American Mathematical Society, vol. 139, no. 5, pp. 16451658, 2011.

[17] Z. Guan and A. G. Kartsatos, "Ranges of generalized pseudomonotone perturbations of maximal monotone operators in reflexive Banach spaces," Contemporary Mathematics, vol. 204, pp. 107-123, 1997.

[18] T. M. Asfaw and A. G. Kartsatos, "A browder topological degree theory for multivalued pseudomonotone perturbations of maximal monotone operators in reflexive Banach spaces," Advances in Mathematical Sciences and Applications, vol. 22, no. 1, pp. 91-148, 2012.

[19] T. M. Asfaw, "New surjectivity results for perturbed weakly coercive operators of monotone type in reflexive Banach spaces," Nonlinear Analysis: Theory, Methods \& Applications, vol. 113, pp. 209-229, 2015.

[20] S. Carl and V. K. Le, "Quasilinear parabolic variational inequalities with multi-valued lower order terms," Zeitschrift für Angewandte Mathematik und Physik, vol. 65, no. 5, pp. 845-864, 2014.

[21] S. Carl, V. K. Le, and D. Motreanu, "Existence, comparison, and compactness results for quasilinear variational-hemivariational inequalities," International Journal of Mathematics and Mathematical Sciences, vol. 2005, no. 3, pp. 401-417, 2005.

[22] S. Carl, "Existence and comparison results for noncoercive and nonmonotone multivalued elliptic problems," Nonlinear Analysis, Theory, Methods and Applications, vol. 65, no. 8, pp. 1532-1546, 2006.

[23] S. Carl and D. Motreanu, "General comparison principle for quasilinear elliptic inclusions," Nonlinear Analysis, Theory, Methods and Applications, vol. 70, no. 2, pp. 1105-1112, 2009.

[24] T. M. Asfaw and A. G. Kartsatos, "New results for perturbations of locally defined generalized pseudomonotone operators in separable reflexive Banach spaces," Advances in Mathematical Sciences and Applications, vol. 24, pp. 1-10, 2014.

[25] T. M. Asfaw, "New variational inequality and surjectivity theories for perturbed noncoercive operators and application to nonlinear problems," Advances in Mathematical Sciences and Applications, vol. 24, pp. 611-668, 2014.

[26] F. E. Browder and B. A. Ton, "Nonlinear functional equations in Banach spaces and elliptic super-regularization," Mathematische Zeitschrift, vol. 105, no. 3, pp. 177-195, 1968.

[27] A. Granas and J. Dugundji, Fixed Point Theory, Springer Monographs in Mathematics, Springer, New York, NY, USA, 2003.

[28] C. P. Gupta, "On an operator equation involving mappings of monotone type," Proceedings of the American Mathematical Society, vol. 53, no. 1, pp. 143-148, 1975.

[29] A. G. Kartsatos, "On the connection between the existence of zeros and the asymptotic behavior of resolvents of maximal monotone operators in reflexive Banach spaces," Transactions of the American Mathematical Society, vol. 350, no. 10, pp. 39673987, 1998.
[30] T. Xiang, "Notes on expansive mappings and a partial answer to Nirenberg's problem," Electronic Journal of Differential Equations, vol. 2013, no. 2, pp. 1-16, 2013.

[31] R. Landes and V. Mustonen, "On pseudo-monotone operators and nonlinear noncoercive variational problems on unbounded domains," Mathematische Annalen, vol. 248, no. 3, pp. 241-246, 1980.

[32] J. Kobayashi and M. Ôtani, "Topological degree for $(S)_{+}$mappings with maximal monotone perturbations and its applications to variational inequalities," Nonlinear Analysis: Theory, Methods \& Applications, vol. 59, no. 1-2, pp. 147-172, 2004.

[33] V. Mustonen, "On elliptic operators in divergence form; old and new with applications," in Proceedings of the International Conference on Function Spaces and Differential Operators and Nonlinear Analysis (FSDONA '04), pp. 188-200, 2004.

[34] D. Kinderlehrer and G. Stampacchia, An Introduction to Variational Inequalities and Their Applications, vol. 88 of Classics in Applied Mathematics, Academic Press, New York, NY, USA, 1980.

[35] Z. Naniewicz and P. Panagiotopoulos, Mathematical Theory of Hemivariational Inequalities and Applications, vol. 188 of Monographs and Textbooks in Pure and Applied Mathematics, Marcel Dekker, New York, NY, USA, 1995.

[36] V. K. Le, "On variational inequalities with maximal monotone operators and multivalued perturbing terms in Sobolev spaces with variable exponents," Journal of Mathematical Analysis and Applications, vol. 388, no. 2, pp. 695-715, 2012.

[37] V. K. Le, "On variational and quasi-variational inequalities with multivalued lower order terms and convex functionals," Nonlinear Analysis: Theory, Methods \& Applications, vol. 94, pp. 12-31, 2014.

[38] S. Carl, V. K. Le, and D. Motreanu, Nonsmooth Variational Problems and their Inequalities: Comparison Principles and Applications, Springer Monographs in Mathematics, Springer, New York, NY, USA, 2007. 


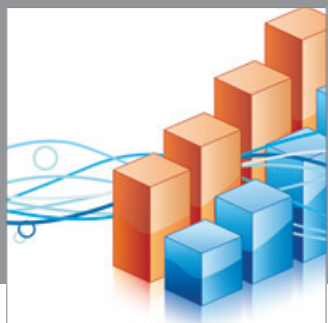

Advances in

Operations Research

mansans

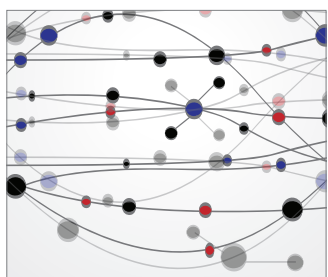

The Scientific World Journal
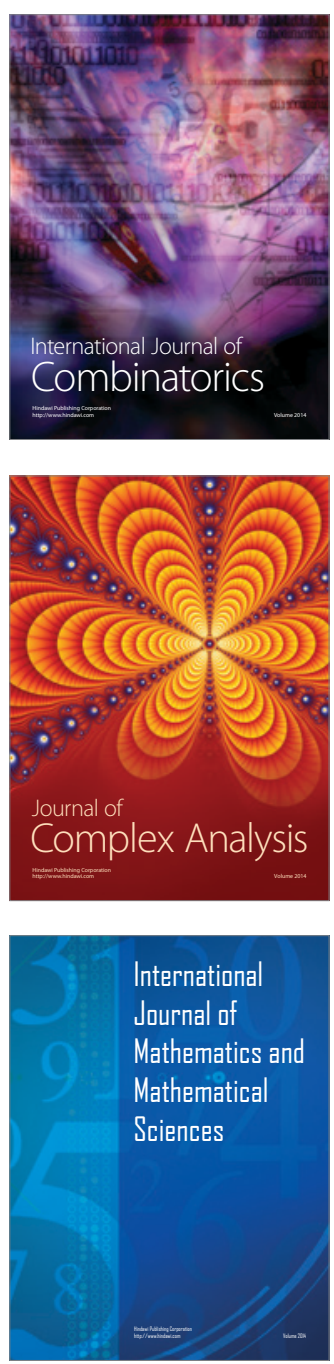
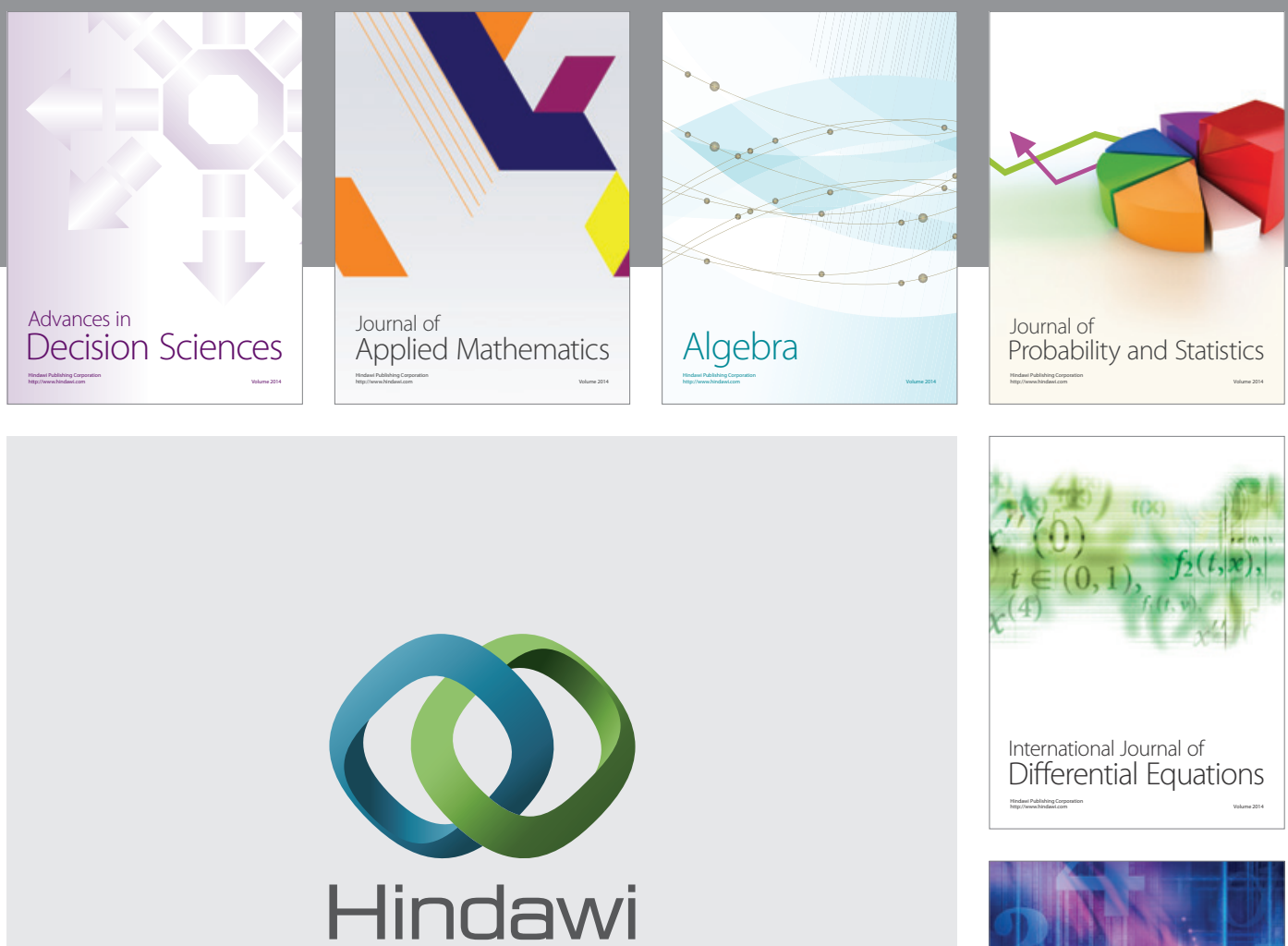

Submit your manuscripts at http://www.hindawi.com
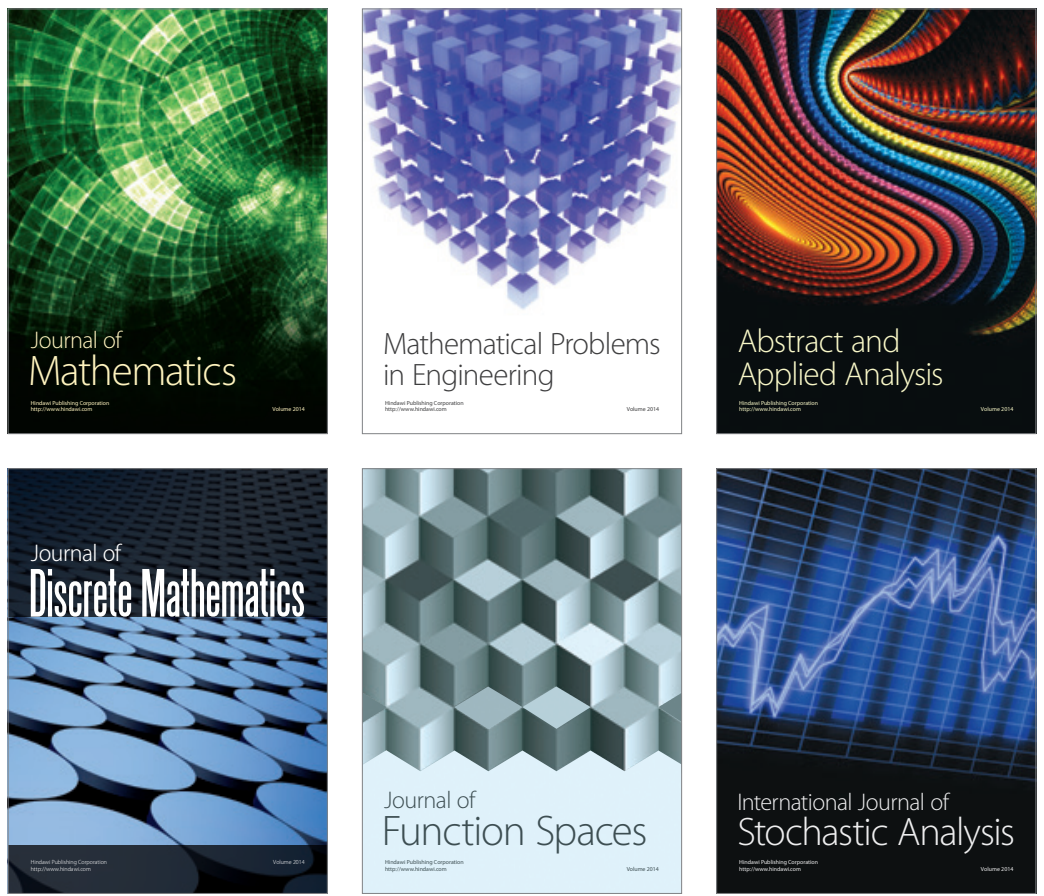

Journal of

Function Spaces

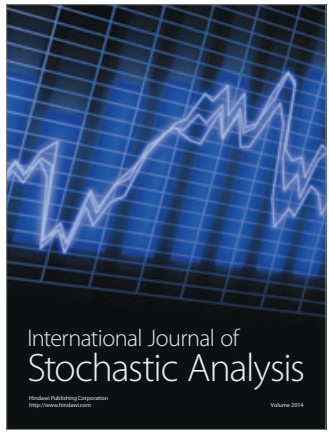

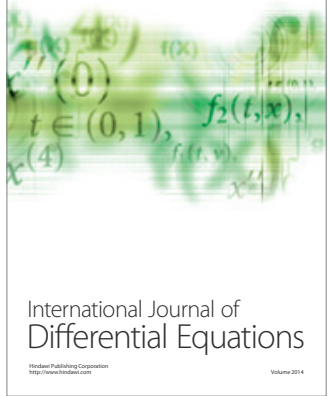
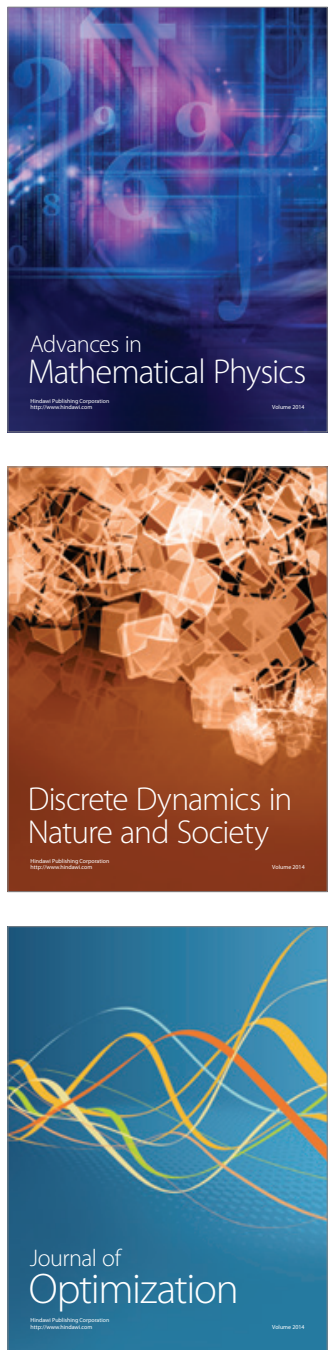